\title{
СИМУЛЯЦИЯ ПРОЦЕССА ВНЕДРЕНИЯ НЕДЕФОРМИРУЕМОГО ИНДЕНТОРА В ДЕФОРМИРУЕМУЮ ПРЕГРАДУ В УСЛОВИЯХ ПЛАСТИЧНОСТИ
}

\section{SIMULATION OF THE INTRUSION OF A NON-DEFORMABLE INDENTER PROCESS INTO DEFPRMABLE BARRIER UNDER CONDITIONS OF PLASTICITY}

\section{O. Yermilova}

Summary. This article simulates the process of deep intrusion of a nondeformable indenter into a deformable barrier with the formation of plastic deformations. The aim of the work is an independent study of the process of plastic deformation of an axisymmetric barrier by an undeformable ball indenter. In the process of numerical mathematical modeling, the following problems were posed and solved: 1) comparing the values of the elastoplastic displacements of the points of the deformable barrier during the intrusion process; 2) comparison of the elastoplastic stresses developed in the process of deep penetration. The work uses the axisymmetric problem of the finite element method, which, as is known, is based on well-known assumptions of the classical theory of elasticity. The results of comparing generated functions of elastoplastic displacements and stresses with the corresponding analogues obtained as a result of using the standard program "Indenter" can be qualified as quite satisfactory.

Keywords: non-deformable ball indenter, deformable cylindrical barrier, elastoplastic displacements, axisymmetric problem, finite element method.

\section{Вве $А$ ение}

4 исленному решению задач статического и динамического моделирования процесса внедрения индентора в деформируемую преграду посвящено в современной литературе большое количество исследований [4-6]. Как известно [4], в такой постановке задача выступает как осесимметричная. Возникающие при этом упруго-пластические деформации относятся к категории больших деформаций. Наиболее эффективной численной стратегией решения данного класса задач является метод маркёров, или метод частиц в ячейках [4], который объединяет в себе все положительные стороны представлений Лагранжа и Эйлера, упреждая при этом проявление какой-либо из их отрицательных сторон [4]. Между тем известно, что в случае оптимальной дискретизации области, при которой исключается

\author{
Ермилова Олеся Викторовна \\ Стариий преподаватель, Донской Государственный \\ Технический Университет \\ olesa-ermilova@mail.ru
}

Аннотация. В статье проводится моделирование процесса глубокого внедрения недеформируемого индентора в деформируемую преграду с образованием пластических деформаций. Целью работы является независимое исследование процесса пластического деформирования осесимметричной преграды недеформируемым шаровым индентором. В процессе численного математического моделирования поставлены и решены следующие задачи: 1) сравнение значений упруго-пластических перемещений точек деформируемой преграды в процессе внедрения; 2) сравнение упруго-пластических напряжений, выработанных в процессе глубокого внедрения. В работе использована осесимметричная задача метода конечных элементов, которая, как известно базируется на общеизвестных предположениях классической теории упругости. Результаты сравнения выработанных функций упруго-пластических перемещений и напряжений с соответствующими аналогами, полученными вследствие применения стандартной программы «Индентор», могут квалифицироваться как вполне удовлетворительные.

Ключевые слова: недеформируемый шаровой индентор, деформируемая цилиндрическая преграда, упруго-пластические перемещения, осесимметричная задача, метод конечных элементов.

возможность перехлёста ячеек, представление Лагранжа обеспечивает наилучшее приближение $[4,6]$.

Применение осесимметричной задачи метода конечных элементов (МКЭ) в перемещениях к численному решению задачи Буссинеска о действии сосредоточенной силы на упругое полупространство [1] обеспечивает превосходное моделирование задачи при известном аналитическом решении. Однако эти результаты ещё не служат основанием для приложения классического МКЭ к решению данного класса задачам в зоне пластичности. В то же время ясно, что в классической постановке МКЭ осесимметричная задача формулируется исключительно в лагранжевом представлении. Однако современные средства информатики и вычислительной техники позволяют осуществлять оптимальные разбиения деформируемой области для дискретных моделей сплошных сред прак- 


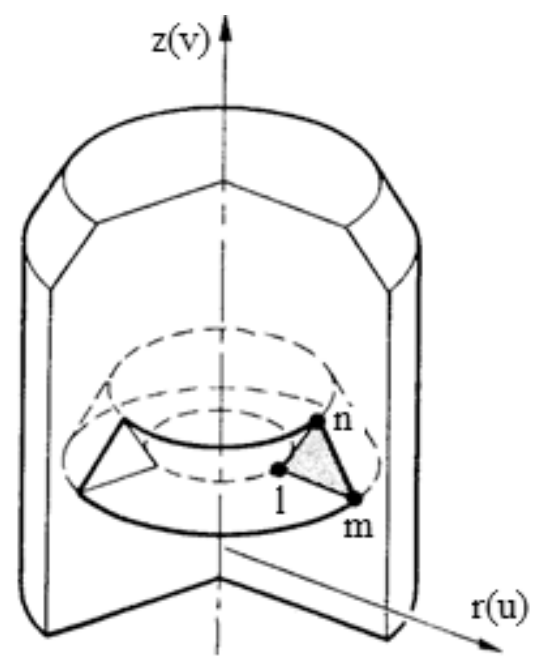

Рис. 1. Расчетная схема осесимметричной задачи

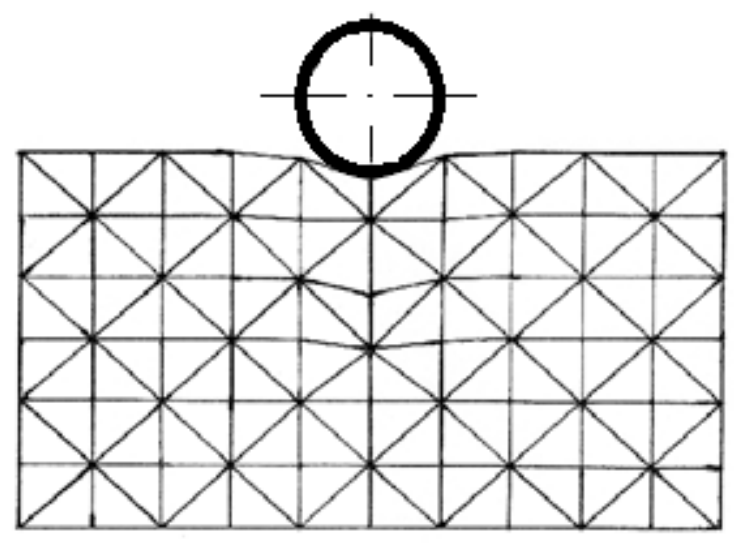

a)

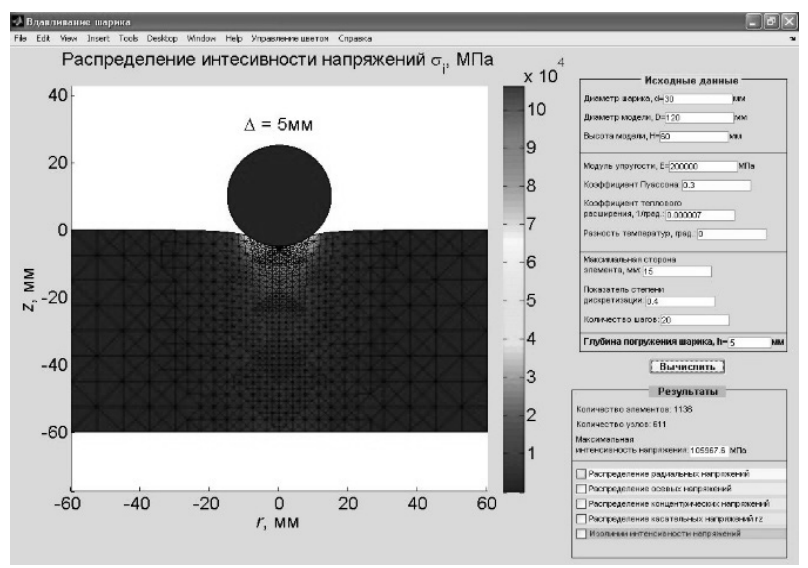

б)

Рис. 3. Деформированные конфигурации препятствия, полученные расчетным кодом (а) и стандартной программой “Индентор” (б)

тически любой размерности. Это обстоятельство делает в настоящее время МКЭ конкурентоспособным по отношению к методу маркёров, использование которого в решении задач больших деформаций сопряжено с необходимостью преодоления более серьёзных трудностей.

В предлагаемой работе представлены результаты статического моделирования посредством традиционного МКЭ задачи внедрения абсолютно твёрдого индентора в деформируемое препятствие.

\section{Формулирование \\ осесимметричной залачи}

Исследование распределения напряжений в телах вращения при осесимметричном нагружении представляет большой практический интерес. Поскольку эти за- дачи также двумерные, то с математической точки зрения они аналогичны задачам о плоском напряжённом и плоском деформированном состояниях. Вследствие симметрии деформированное и напряжённое состояния в любом сечении по оси симметрии тела полностью определяются двумя компонентами перемещений [1].

Перемещения каждого узла конечного элемента Imn, например I, имеют две компоненты (рис. 1):

$$
\left\{\mathbf{U}_{\mathrm{i}}^{\mathrm{l}}\right\}=\left\{\begin{array}{c}
\mathrm{U}_{1}^{1} \\
\mathrm{U}_{2}^{1}
\end{array}\right\}=\left\{\begin{array}{l}
\mathrm{U}^{1} \\
\mathrm{~V}^{1}
\end{array}\right\},
$$

откуда следует, что вектор узловых перемещений элемента определяется следующим шестимерным вектор-столбцом: 


$$
\{\mathbf{U}\}=\left\{\begin{array}{c}
\mathbf{U}_{\mathrm{i}}^{1} \\
\mathbf{U}_{\mathrm{i}}^{\mathrm{m}} \\
\mathbf{U}_{\mathrm{i}}^{\mathrm{n}}
\end{array}\right\}, \quad \mathrm{i}=1,2 .
$$

Для рассматриваемой плоской задачи МКЭ перемещения u и v принимаются линейными относительно $\mathrm{x}_{\mathrm{j}}$ [2]:

$$
u_{i}=\alpha_{i}+\beta_{i j} x_{j}, \quad i, j=1,2,
$$

где коэффициенты $\alpha_{\mathrm{i}}$ и $\beta_{\mathrm{ij}}$ постоянны в пределах каждого элемента.

\section{Численная \\ реализачия захачи}

В качестве примера численного моделирования осесимметричной задачи МКЭ приводится решение задачи определения больших упруго-пластических деформаций вследствие приложения сосредоточенной силы на упруго-пластичное полупространство, представляющее собой однородную и изотропную сплошную среду. Материалом среды принята незакаленная СТ-45 $(\mathrm{E}=200$ $\Gamma П а,\left[\sigma_{v}=363 \mathrm{MПa}\right]$ и v = 0.3).
Габаритные размеры осесимметричной деформиру-

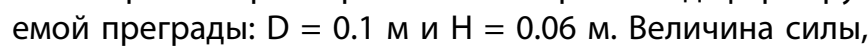
приложенной со стороны шарового недеформируемого индентора диаметра в $=0.03 \mathrm{M},-\mathrm{F}=160 \mathrm{MH}$; число этапов численного воспроизведения больших упругопластических деформаций $-\mathrm{NES}=500$

Расчетные результаты проведенного тестирования хорошо согласуются с итогами численного решения той же задачи, полученными с помощью стандартной программы “Индентор" (рис. 3, а и б), основанной также на неизменном формализме осесимметричной задачи методе конечных элементов.

\section{Зак^ючение}

Представлены результаты численного моделирования больших упруго-пластических деформаций на основе численного решения осесимметричной задачи МКЭ. Сравнительный анализ расчётных характеристик (перемещений, напряжений и деформаций), выработанных на основе составленной программы и прикладного пакета "Индентор" соответственно убеждает в хорошей аппроксимации задачи при использовании относительно небольшого количества конечных элементов и узлов аппроксимации.

1. Зенкевич 0., Метод конечных элементов в технике.— М.: Наука, 1975.

2. Демидов С.П., Теория упругости.-М.: Наука, 1979.

3. Тимошенко С.П., Гудьер Дж. Теория упругости.-М.: Наука, 1975.

4. Гриднеева В.А., Корнеев А. И., Трушков В. Г. Численный расчет напряженного состояния и разрушения плиты конечной толщины при ударе бойками различной формы // Изв. РАН. МТТ. № 1, 1977.—C. 146-157.

5. Багдоев А.Г., Ванцян А. А. Проникание тонких тел в упругие среды // Изв. АН Арм. ССР. Механика. — 1981.— Т. 3.— 4, № 1.— С. 3-15.

6. Ванцян А.А., Овсепян 0. Х. Динамическое взаимодействие деформируемого индентора и преграды при наличии разрядного тока.- - Ереван: Авт. изд.-во, 2010,299 c. 